\title{
REGULARITY RESULTS FOR A CLASS OF SEMILINEAR PARABOLIC DEGENERATE EQUATIONS AND APPLICATIONS*
}

\author{
MARCO PAPI ${ }^{\dagger}$
}

\begin{abstract}
We prove some regularity results for viscosity solutions to strongly degenerate parabolic semilinear problems. These results apply to a specific model used for pricing MortgageBacked Securities and allow a complete justification of the existence of the proposed risk-neutralmeasure.
\end{abstract}

\section{Introduction}

In this paper we investigate the second order regularity of bounded viscosity solutions to the semilinear parabolic equation of degenerate type

$$
\partial_{t} u+H\left(x, t, u, \nabla u, \nabla^{2} u\right)=0
$$

where the Hamiltonian function is given by

$$
\begin{aligned}
H(x, t, u, p, X)= & -\frac{1}{2} \operatorname{tr}\left(\sigma(t) \sigma^{\top}(t) X\right)+\langle\mu(x, t), p\rangle+\lambda(u)\left|\sigma^{\top}(t) p\right|^{2} \\
& +\eta(u)\left\langle\sigma^{\top}(t) p, w(x, t)\right\rangle+f(x, t, u),
\end{aligned}
$$

for $(x, t, u, p, X) \in \mathbb{R}^{N} \times(0, T) \times(a, b) \times \mathbb{R}^{N} \times \mathcal{S}^{N}$, where $(a, b) \subset \mathbb{R}$ is a given interval (possibly unbounded) and $\mathcal{S}^{N}$ is the space of $N \times N$ symmetric matrices. In (1.2) $\sigma(t)$ is a $N \times d$ matrix, with $N \geq d$, while $\mu(x, t), w(x, t)$ belong to $\mathbb{R}^{N}$ and $\mathbb{R}^{d}$ respectively, $\lambda, \eta, f$ are real-valued functions, $\operatorname{tr}$ denotes the trace of a square matrix and $(\cdot)^{\top}$ denotes the transpose. The motivation for considering Hamiltonians of this form comes from a problem in mathematical finance which we describe in the sequel. Observe that the matrix $\sigma \sigma^{\top}$ has rank at most $d$ and so equation (1.1) is a strongly degenerate parabolic if $d<N$, which is the interesting case for our application. We study our equation in the framework of viscosity solutions of Crandall and Lions [6]. Let us observe that the Hamiltonian in (1.2) does not satisfy the standard assumptions that are made in the theory of viscosity solutions. In fact, one usually assumes some monotonicity condition on the Hamiltonian with respect to $u$, e.g. the existence of a constant $\theta \geq 0$ such that

$$
H(x, t, u, p, X) \geq H(x, t, v, p, X)-\theta(u-v), \quad \forall u>v,
$$

which is not satisfied by the Hamiltonian in (1.2). Therefore, even the existence of a viscosity solution to our equation does not follow from the standard theory. However, our Hamiltonian has some good features which can be used to compensate for the lack of monotonicity with respect to $u$, and so in a previous paper [18] we have proved that the Cauchy problem associated with (1.1) and (1.2) possesses a unique viscosity solution $u$, which is Lipschitz continuous with respect to $x$.

Here we prove further regularity properties: under suitable assumptions on the coefficients, we show that $u$ is actually $W^{2, \infty}$ with respect to $x$ and Lipschitz continuous in $t$. This regularity result is stronger than the usual ones known for equations of the form (1.1) and has important consequences for our application to mathematical

\footnotetext{
*Received: June 20, 2002; accepted (in revised version): December 24, 2002.

†Istituto per le Applicazioni del Calcolo "M.Picone", V.le del Policlinico 137, I-00161 Roma, Italy and Dipartimento di Matematica di Roma "Tor Vergata", Via della Ricerca Scientifica, 00133, Italy
} 
finance. We point out that the particular structure of the Hamiltonian in (1.2) plays a crucial role in our analysis and it should not be expected that the same results hold for a general degenerate parabolic equation.

Our main result (Theorem 2.4) about the $W^{2, \infty}$-regularity of the solution $u$ to equation (1.1) is obtained showing that $u$ is both semiconvex and semiconcave (see section 2 for the definition). These properties are familiar in the theory of HamiltonJacobi equations, both of first and second order. In many cases, one is able to prove the semiconvexity (resp. semiconcavity) of the solution under the requirement that the Hamiltonian $H$ is concave (resp. convex) with respect to $p$. Therefore, it is usually hard to show that the two properties hold simultaneously, except of course in the case when the equation is strictly parabolic. Our method of proof relies on a change of unknown which transforms the original equation (1.1) in an equivalent one where the Hamiltonian satisfies some additional structural properties. Using these properties we are able to prove the semiconvexity of the solution of the transformed equation, which implies the semiconvexity of the original solution $u$. By a similar argument, using a different transformation, we obtain the semiconcavity of $u$, and the two properties together yield the $W^{2, \infty}$-regularity.

We recall that Ishii and Lions [12] have proved the semiconcavity of the solution for the Bellman equations, but the class of nonlinearities they consider does not include our Hamiltonian (1.2). Another related result is the one by Giga, Goto, Ishii and Sato, [9], who have proved the convexity preserving property of the equation. However, their technique cannot be applied in our case because it is not suitable for treating equations where the Hamiltonian depends on $u$.

Our results can be applied to a semilinear equation (see (2.3)) proposed in [17] as a differential model for pricing some widely traded American financial instruments, the Mortgage-Backed Securities $(M B S)$; the model was derived following the outline of X. Gabaix in [8].

Although we shall not study here the financial issues coming from this model, in section 2 we give a concise discussion of the financial model analyzing the particular structure of the equation.

The rest of the paper is organized as follows: in section 3, we introduce the notation that will be used throughout this paper and we state our results. In section 4 we give the detailed proofs of these. In section 5 we discuss the time regularity, and we show that $u$ is Lipschitz continuous in time.

\section{Financial motivations: the MBS model}

Recently, the theory about financial markets, the mathematical frameworks for modelling them, and arbitrage theory have reached a high development and have taken a prominent position in the mathematical literature (we refer the interested reader to some reference books dealing with these arguments, like [7], and [15]). However, some financial derivatives, like MBSs, and still need of specific models which are conformed to their peculiarities.

MBSs are the products of a securitization of pools of mortgages. Every mortgageholder in a pool holds the right to prepay her debt at every time between 0 and the maturity $T$. This American-style option determines the stochasticity in the valuation of the price of a Mortgage-Backed from that pool. Many factors affect prepayments and this creates a remarkable complexity for generating forecasts.

The arbitrage pricing principle applies to financial instruments whose cash flows are related to the values of economic factors, such as the interest rates. It implies that the price $V_{t}$ of a MBS can be expressed as $V_{t}=h\left(X_{t}, T-t\right)+U\left(X_{t}, T-t\right)$, 
where $h$ contains the information about prepayments and $U$ is given by a conditional expectation up to the time $t$, w.r.t. a particular probability measure $Q$ :

$$
U\left(X_{t}, T-t\right)=\mathbb{E}_{t}^{Q}\left[\int_{t}^{T}(\tau-r(T-s)) e^{-\int_{t}^{s} r(T-\kappa) d \kappa} h\left(X_{s}, T-s\right) d s\right], \text { a.s, }(2.1
$$

where $\tau>0$ is a constant and $r$ is a deterministic function. The process $X_{t}$, which describes the economic factors affecting the security price is a $N$-dimensional Ito process given by the dynamics

$$
d X_{t}=\mu\left(X_{t}, T-t\right) d t+\sigma(T-t) d W_{t}, \quad 0<t \leq T,
$$

where $W_{t}$ denotes a $d$-dimensional standard Brownian Motion over the probability space $\Omega$.

Assuming the model for $Q$, proposed by X.Gabaix and O.Vigneron in [8], under suitable conditions, computing the expectation (2.1) reduces to the solution of the following partial differential equation:

$$
\partial_{t} U-\frac{1}{2} \operatorname{tr}\left(\sigma \sigma^{\top} \nabla^{2} U\right)-\langle\mu, \nabla U\rangle+\rho \frac{\left|\sigma^{\top} \nabla U\right|^{2}}{U+h+\xi}+r(U+h)-\tau h=0,
$$

in $\mathbb{R}^{N} \times(0, T)$, where $U(x, 0)=0, \rho>0$ is a constant and $\xi=\xi(t), h=h(x, t) \geq 0$ are smooth functions. Observe that if $U$ is a viscosity solution of (2.3), then $u=U+h+\xi$ solves equation (1.1) with a Hamiltonian of the form (1.2).

The incompleteness of the market implies the choice of a market price of risk (m.p.r) (see also [15]), and via the Girsanov's Theorem [14] this corresponds to the specification of a new probability measure $(Q)$.

Actually, when the market is not complete, the m.p.r is not unique and it is chosen by data analysis; this approach is also followed in [8]: In fact, they endogenously assign a form for the m.p.r related to a MBS and they motivate this choice by a statistical argument, but the mathematical toolbox that would required to rigorously derive their results is still largely to be developed.

A characteristic of equation (2.3) is the strong degeneracy. In fact, also this feature comes from the dependence of the security payoff on the trajectory followed by one or more of the underlying Markovian processes $\left(X_{t}\right)$. We illustrate this point with a simple consideration. One possible index $\left(b_{t}\right)$ of the incentive to prepay a mortgage is the amount by which a particular interest rate $\bar{r}_{s}$ is below some given level $\bar{r}$. The factor is represented by $b_{t}=\int_{0}^{t} \mu\left(\bar{r}-\bar{r}_{s}\right) d s$, where $\mu$ is an increasing function, and this form gives the absence of diffusion in the $b$ direction.

The appeal to differential equations in financial modelling has become a standard approach, and in many cases it represents the best way for valuing derivatives. Therefore the study of solutions of the pricing equations of more complex financial instruments and their numerical solutions has become an important technique available to practitioners of modern quantitative finance. However, up to now the $P D E$ approach for the valuation of MBSs with the additional specification of a nonlinear form for the m.p.r was not followed. Although equation (2.3) represents a reduced version of a more general situation which includes a model of the issuance of future securities, the model outlines a new manner for treating MBSs derivatives. The existence, uniqueness and regularity properties for the solution of that equation holds an essential role: actually, it is well known that an arbitrary specification of the m.p.r. may lead to arbitrage opportunities; hence, the existence of a solution for (2.3) is the proof that there exists a new risk-neutral market measure through evaluating these securities. 


\section{Main Results}

In this section, we fix some notations and present our main results about the regularity of the solution, sections 4 and 5 being devoted to their proofs.

We denote by $\mathcal{M}_{m, n}(\mathbb{R})$ the space of matrices with real coefficients and $m$ rows and $n$ columns, with the usual norm

$$
\|A\|=\sup _{x \in \mathbb{R}^{n}:|x|=1}|A x|, \quad \forall A \in \mathcal{M}_{m, n}(\mathbb{R}) .
$$

Moreover we denote by $\operatorname{Im}(A)$ the range of the linear map defined by the matrix $A$. If $g: \mathbb{R}^{N} \times[0, T) \times I \rightarrow \mathbb{R}$, where $I$ is a bounded interval in $\mathbb{R}$, then we set

$$
\|g(t)\|_{\infty}=\operatorname{essup}_{(x, u) \in \mathbb{R}^{N} \times I}|g(x, t, u)|, \quad \forall t \in[0, T),
$$

while $\|g\|_{\infty}$ denotes as usual the essential supremum of $g$ over all variables. We use the standard notation for Sobolev spaces denoting with $\mathbb{W}^{k, \infty}$ the space of functions which are bounded, together with their weak derivatives up to the order $k$. If a function depends on more than one group of variables, we use distinct superscripts to allow for a different number of derivatives w.r.t. the different arguments. For instance, we denote by $\mathbb{W}^{2,1, \infty}\left(\mathbb{R}^{N} \times(0, T)\right)$ the space of bounded functions $u$, with weak derivatives $\partial_{t} u, \partial_{i} u, \partial_{i j}^{2} u \in L^{\infty}\left(\mathbb{R}^{N} \times(0, T)\right)$, for $i, j=1, \ldots, N$, endowed with the norm

$$
\|u\|_{\mathbb{W}^{2,1, \infty}}=\left\|\partial_{t} u\right\|_{\infty}+\|\nabla u\|_{\infty}+\left\|\nabla^{2} u\right\|_{\infty} .
$$

Let us now recall the definition of semiconvex and semiconcave functions.

Definition 3.1. A function $g$ in $\mathbb{R}^{N}$ is semiconvex with constant $L>0$, if

$$
g(x+h)+g(x-h)-2 g(x) \geq-L|h|^{2},
$$

for every $x, h \in \mathbb{R}^{N}$. We say that $g$ is semiconcave with constant $L>0$ if $-g$ is semiconvex with constant $L$.

It is easy to see that $g$ is semiconvex if and only if it satisfies $\partial_{\nu \nu} g \leq L$ in the sense of distributions for all $\nu \in \mathbb{R}^{n}$ such that $|\nu|=1$, and that $g \in W^{2, \infty}\left(\mathbb{R}^{N}\right)$ if and only if $g$ is bounded and is both semiconcave and semiconvex.

In the following, we study the regularity of the viscosity solution of equation (1.1) with a given continuous and bounded initial datum $u_{0}$. The coefficients $\mu, \lambda, \eta, f$ in the equation are assumed to be at least continuous and hereafter $\sigma$ is continuous in $[0, T]$. We omit the definition of a viscosity sub/super solution of a differential problem like (1.1), and refer the reader to some classic works about viscosity theory, such as [6] or [12]. From our comparison result in [18], standard techniques (see e.g. [6]) can be used to deduce the existence of a unique viscosity solution for the equation (1.1), taking values in the interval $(a, b)$. Moreover, using the same arguments of Theorem 4.5 in [18], it can also be deduced that, if $\mu, \lambda, \eta, f$ and $u_{0}$ are Lipschitz continuous with respect to $x$ uniformly in $t$, then the solution $u$ has the same regularity. Therefore, we assume in the following the existence of a viscosity solution $u$ of (1.1), such that $u$ takes values in a bounded interval $I \subset(a, b), u(\cdot, t) \in \mathbb{W}^{1, \infty}\left(\mathbb{R}^{N}\right)$ and whose norm is uniformly bounded for $t \in[0, T)$. Starting from this, we are able to prove the following results.

THEOREM 3.2. Let the viscosity solution $u$ of the equation (1.1) take values in a bounded closed interval $I \subset(a, b)$, and let $\mu(\cdot, t), w(\cdot, t) \in \mathbb{W}^{2, \infty}\left(\mathbb{R}^{N}\right), f(\cdot, t, \cdot) \in$ $\mathbb{W}^{2, \infty}\left(\mathbb{R}^{N} \times I\right)$, uniformly in time. Assume: 
i) $\lambda \in \mathcal{C}((a, b)), \eta \in \mathcal{C}((a, b)) \cap \mathcal{C}^{2}(I)$;

ii) for every $(x, t) \in \mathbb{R}^{N} \times(0, T), w(x, t) \in \operatorname{Im}\left(\sigma^{\top}(t)\right)$.

If $u_{0}$ is semiconvex, then there are positive constants $C, M_{0}, c_{0}$, such that

$$
u(x+h, t)+u(x-h, t)-2 u(x, t) \geq-\left(M_{0} e^{C t}+c_{0}\right)|h|^{2},
$$

holds for every $x, h \in \mathbb{R}^{N}$, and $t \in(0, T)$. Therefore, for every $t \in(0, T), u(\cdot, t)$ is semiconvex.

We have also the analogous result for the semiconcavity of $u$.

THEOREM 3.3. Let the viscosity solution $u$ of the equation (1.1) take values in a bounded closed interval $I \subset(a, b)$, and let $\mu(\cdot, t), w(\cdot, t) \in \mathbb{W}^{2}, \infty\left(\mathbb{R}^{N}\right), f(\cdot, t, \cdot) \in$ $\mathbb{W}^{2, \infty}\left(\mathbb{R}^{N} \times I\right)$, uniformly in time. Assume:

i) $\lambda \in \mathcal{C}((a, b)), \eta \in \mathcal{C}((a, b)) \cap \mathcal{C}^{2}(I)$;

ii) for every $(x, t) \in \mathbb{R}^{N} \times(0, T), w(x, t) \in \operatorname{Im}\left(\sigma^{\top}(t)\right)$.

If $u_{0}$ is semiconcave, then there are positive constants $C, M_{0}, c_{0}$, such that

$$
u(x+h, t)+u(x-h, t)-2 u(x, t) \leq\left(M_{0} e^{C t}+c_{0}\right)|h|^{2},
$$

holds for every $x, h \in \mathbb{R}^{N}$, and $t \in(0, T)$. Therefore, for every $t \in(0, T), u(\cdot, t)$ is semiconcave.

As a corollary, we deduce the announced result about the $W^{2, \infty}$ regularity of $u$.

THEOREM 3.4. If $\mu, w, f, \lambda$ and $\eta$ satisfy the assumptions of Theorems 3.2 and 3.3 and $u_{0} \in \mathbb{W}^{2, \infty}\left(\mathbb{R}^{N}\right)$, then $u(t) \in \mathbb{W}^{2, \infty}\left(\mathbb{R}^{N}\right)$, uniformly in time.

In addition, we have the following result about the time regularity.

Theorem 3.5. If $\mu, w, f, \lambda$ and $\eta$ satisfy the assumptions of Theorems 3.2 and 3.3 and $u_{0} \in \mathbb{W}^{2, \infty}\left(\mathbb{R}^{N}\right)$, then

$$
u \in \mathbb{W}^{2,1, \infty}\left(\mathbb{R}^{N} \times(0, T)\right) .
$$

In section 4, we first prove a preliminary version of Theorems 3.2 and 3.3, where some additional structural and regularity assumptions are imposed on the function $\lambda$ (see Propositions 4.1 and 4.2). Then, these conditions on $\lambda$ can be removed by exploiting a particular compatibility between the second order linear term and the first order term in the equation (1.1). This latter step is based on the well-known property that the notion of viscosity solution is invariant under a global and increasing change of the variable $u$.

\section{Proof of the Results}

This part is devoted to the presentation of the technical results which are useful for proving Theorems 3.2, 3.3 and 3.4, illustrated in the previous section.

Proposition 4.1. Consider a viscosity solution u of problem (1.1) valued in a bounded closed subinterval $I$ of the domain $(a, b)$, such that $u(\cdot, t) \in \mathbb{W}^{1, \infty}\left(\mathbb{R}^{N}\right)$, with a norm uniformly bounded for $t \in[0, T)$. Assume a semiconvex initial datum $u_{0}$ (see definition 3.1) with a constant $L_{0}>0$. Suppose that, $\mu(\cdot, t), w(\cdot, t)$ are $\mathbb{W}^{2, \infty}\left(\mathbb{R}^{N}\right)$ functions and, $f(\cdot, t, \cdot) \in \mathbb{W}^{2, \infty}\left(\mathbb{R}^{N} \times I\right)$, uniformly in time. Moreover, assume:

i) $\lambda, \eta \in \mathcal{C}((a, b)) \cap \mathcal{C}^{2}(I)$;

ii) $\lambda<0, \lambda^{\prime}>0, \lambda \lambda^{\prime \prime}-2\left(\lambda^{\prime}\right)^{2}>0$, over $I$

iii) for every $(x, t) \in \mathbb{R}^{N} \times(0, T)$, w $(x, t) \in \operatorname{Im}\left(\sigma^{\top}(t)\right)$. 
Then, there are positive constants $C$, which depends on $\sup _{t \in[0, T)}\|u(t)\|_{\mathbb{W}_{11, \infty}}$ and $c_{0}$, which depends on $L_{0}$ and $\operatorname{Lip}\left(u_{0}\right)$, such that

$$
u(x+h, t)+u(x-h, t)-2 u(x, t) \geq-c_{0} e^{C t}|h|^{2}
$$

holds for every $(x, h, t) \in \mathbb{R}^{N} \times \mathbb{R}^{N} \times(0, T)$. In particular $u(\cdot, t)$ is semiconvex, for every $t \in(0, T)$.

The equivalent result for the semiconcave property of the solution, is the following.

Proposition 4.2. Consider a viscosity solution u of problem (1.1) valued in a bounded closed subinterval $I$ of the domain $(a, b)$, such that $u(\cdot, t) \in \mathbb{W}^{1, \infty}\left(\mathbb{R}^{N}\right)$, with a norm uniformly bounded for $t \in[0, T)$. Assume a semiconcave initial datum $u_{0}$ (see definition 3.1) with a constant $L_{0}>0$. Suppose that, $\mu(\cdot, t), w(\cdot, t)$ are $\mathbb{W}^{2, \infty}\left(\mathbb{R}^{N}\right)$ functions and, $f(\cdot, t, \cdot) \in \mathbb{W}^{2, \infty}\left(\mathbb{R}^{N} \times I\right)$, uniformly in time. Moreover assume:

i) $\lambda, \eta \in \mathcal{C}((a, b)) \cap \mathcal{C}^{2}(I)$;

ii) $\lambda>0, \lambda^{\prime}>0, \lambda \lambda^{\prime \prime}-2\left(\lambda^{\prime}\right)^{2}>0$, over $I$;

iii) for every $(x, t) \in \mathbb{R}^{N} \times(0, T), w(x, t) \in \operatorname{Im}\left(\sigma^{\top}(t)\right)$.

Then, there are positive constants $C$, which depends on $\sup _{t \in[0, T)}\|u(t)\|_{\mathbb{W} 1, \infty}$ and $c_{0}$, which depends on $L_{0}$ and $\operatorname{Lip}\left(u_{0}\right)$, such that

$$
u(x+h, t)+u(x-h, t)-2 u(x, t) \leq c_{0} e^{C t}|h|^{2}
$$

holds for every $(x, h, t) \in \mathbb{R}^{N} \times \mathbb{R}^{N} \times(0, T)$. In particular $u(\cdot, t)$ is semiconcave, for every $t \in(0, T)$.

REMARK 4.3. We can observe the analogies between the two formulations 4.1, 4.2. Actually the hypothesis $\lambda^{\prime}>0$ is the same in both of the Propositions. This can be interpreted as a consequence of the comparison principle, which requires a monotonicity for the Hamiltonian w.r.t. $u$ (see [9], p. 462). Meanwhile of course, the other expressions in $i i$ ) have exactly an opposite sign, showing a perfect symmetry between the two formulations.

The proof of Proposition 4.2 follows by a similar argument as for Proposition 4.1, so it is omitted. The proof is based on the same technique proposed in [12], which uses a particular test function. Nevertheless, the nonlinear part in equation (1.1) does not allow to close the proof as in that work. Hence, we turn to a semiconcavity property of the nonlinear part with respect to $(u, \nabla u)$, and to a monotonicity with respect to the unknown.

Remark 4.4. We observe that if a function $g$ is semiconvex, as in the Definition 3.1, and is Lipschitz continuous with constant $\operatorname{Lip}(g)$, then for all $x, y, z$, the following inequality holds:

$$
g(x)+g(y)-2 g(z) \geq-L\left(|x-z|^{2}+|y-z|^{2}\right)-\operatorname{Lip}(g)|x+y-2 z| .
$$

Proof of Proposition 4.1. Consider the function $v=u e^{-C t}$, where $C$ is a nonnegative constant. Then $v$ is an $x$-Lipschitz continuous function uniformly w.r.t. the time $t \in[0, T)$. Moreover $v$ is a continuous viscosity solution of the following equation:

$$
\begin{aligned}
\partial_{t} v & -\frac{1}{2} \operatorname{tr}\left(\sigma \sigma^{\top} \nabla^{2} v\right)+\langle\mu, \nabla v\rangle+\lambda\left(v e^{C t}\right) e^{C t}\left|\sigma^{\top} \nabla v\right|^{2} \\
& +\eta\left(v e^{C t}\right)\left\langle\sigma^{\top} v, w\right\rangle+e^{-C t} f\left(x, t, v e^{C t}\right)+C v=0,
\end{aligned}
$$


where $(x, t) \in \mathbb{R}^{N} \times(0, T)$, and $v_{0}=u_{0}$. Now, we go to prove that, under the assumptions made on $\lambda$ and $\eta$, the result is the semiconvexity of the function $v$, and therefore semiconvexity of the function $u$. In particular, we are going to show that,

$$
v(x, t)+v(y, t)-2 v(z, t) \geq-M\left(|x-z|^{4}+|y-z|^{4}+|x+y-2 z|^{2}\right)^{\frac{1}{2}}
$$

for every $x, y, z \in \mathbb{R}^{N}, t \in[0, T)$, where $M=\frac{\sqrt{3}}{2} \max \left(L_{0}, \operatorname{Lip}\left(u_{0}\right)\right)$. By the assumptions on $u$, the initial datum is Lipschitz continuous. Therefore, this constant is well defined; this obviously yields the assertion on $v$ by setting $x=z+h$ and $y=z-h$. It is easy to see that the above inequality is equivalent to the following one:

$$
v(x, t)+v(y, t)-2 v(z, t) \geq-M\left[\delta+\frac{1}{\delta}\left(|x-z|^{4}+|y-z|^{4}+|x+y-2 z|^{2}\right)\right],
$$

for every $x, y, z \in \mathbb{R}^{N}$ and for every $\delta>0$. Hence, fix $\varepsilon, \delta, \gamma>0$, and consider the following test function:

$$
\begin{aligned}
& \Psi(x, y, z, t)=v(x, t)+v(y, t)-2 v(z, t)+M\left[\delta+\frac{1}{\delta}\left(|x-z|^{4}\right.\right. \\
& \left.\left.+|y-z|^{4}+|x+y-2 z|^{2}\right)\right]+\varepsilon|x|^{2}+\varepsilon|y|^{2}+\varepsilon|z|^{2}+\frac{\gamma}{T-t}
\end{aligned}
$$

defined for $(x, y, z, t) \in D=\mathbb{R}^{3 N} \times[0, T)$. The assertion (4.5) is equivalent to prove that, for every $\delta, \gamma>0$, there exists $\varepsilon_{0}=\varepsilon_{0}(\delta, \gamma)>0$, such that for every $0<\varepsilon<\varepsilon_{0}$, the following holds true:

$$
\inf _{D} \Psi \geq 0
$$

Actually, if (4.7) holds, then fixing a point of $D$, we send $\varepsilon$ to zero in the inequality $\Psi \geq$ 0 , and then send also $\gamma$ to zero obtaining (4.5). So we limit ourselves to considering the assertion (4.7).

We assume as usual that (4.7) is false, and will get a contradiction. Therefore, there exist $\delta_{0}, \gamma_{0}>0$ and a sequence $\varepsilon_{j} \rightarrow 0$, as $j \rightarrow \infty$, such that

$$
\inf _{D} \Psi<0
$$

with $\delta=\delta_{0}, \gamma=\gamma_{0}$, and $\varepsilon=\varepsilon_{j}$, for every integer $j>0$. Consider a minimizing sequence $\left(x_{k}, y_{k}, z_{k}, t_{k}\right) \in D$ for $\Psi$. By (4.8), the definition (4.6) and the boundedness of $v$, we see that $\left(x_{k}, y_{k}, z_{k}\right)$ must be bounded, so we can extract a convergent subsequence, which converges to some point $(\widehat{x}, \widehat{y}, \widehat{z}, \widehat{t}) \in D$, which, by the continuity of $v$, is a global minimum point for $\Psi$ over $D$; moreover, $\widehat{t}$ (which is obviously less than $T$ ) is strictly positive. In fact, if $\widehat{t}=0$, by (4.8) and the Remark 4.4, we obtain.

$$
\begin{aligned}
0> & \Psi(\widehat{x}, \widehat{y}, \widehat{z}, 0) \geq \\
\geq & u_{0}(\widehat{x})+u_{0}(\widehat{y})-2 u_{0}(\widehat{z})+M\left[\delta+\frac{1}{\delta}\left(|\widehat{x}-\widehat{z}|^{4}+|\widehat{y}-\widehat{z}|^{4}+|\widehat{x}+\widehat{y}-2 \widehat{z}|^{2}\right)\right] \geq \\
\geq & -L_{0}\left(|\widehat{x}-\widehat{z}|^{2}+|\widehat{y}-\widehat{z}|^{2}\right)-\operatorname{Lip}\left(u_{0}\right)|\widehat{x}+\widehat{y}-2 \widehat{z}|+ \\
& +M\left[\delta+\frac{1}{\delta}\left(|\widehat{x}-\widehat{z}|^{4}+|\widehat{y}-\widehat{z}|^{4}+|\widehat{x}+\widehat{y}-2 \widehat{z}|^{2}\right)\right] \geq \\
& \quad\left[-L_{0}+\frac{2}{\sqrt{3}} M\right]\left(|\widehat{x}-\widehat{z}|^{2}+|\widehat{y}-\widehat{z}|^{2}\right)+\left[-\operatorname{Lip}\left(u_{0}\right)+\frac{2}{\sqrt{3}} M\right]|\widehat{x}+\widehat{y}-2 \widehat{z}| \geq 0 .
\end{aligned}
$$


Hence, by the previous contradiction, we deduce that $\widehat{t}>0$. So the minimum point is an interior "stationary" point of $\Psi$. Setting the functions

$$
\begin{aligned}
g(x, y, z, t)= & -v(x, t)-v(x, t)+2 v(z, t) \\
\Phi(x, y, z, t)= & M\left[\delta+\frac{1}{\delta}\left(|x-z|^{4}+|y-z|^{4}+|x+y-2 z|^{2}\right)\right] \\
& +\varepsilon|x|^{2}+\varepsilon|y|^{2}+\varepsilon|z|^{2}+\frac{\gamma}{T-t}
\end{aligned}
$$

the result is that $g-\Phi=-\Psi$ has a global interior maximum point at $(\widehat{\xi}, \widehat{t})=(\widehat{x}, \widehat{y}, \widehat{z}, \widehat{t})$. Therefore, we can apply the classical Theorem of M.G. Crandall and H. Ishii, in [5], about the maximum principle for semicontinuous functions to deduce that for $\kappa=\frac{1}{\varepsilon}>0$, there exist $\left(b_{i}, X_{i}\right) \in \mathbb{R} \times \mathcal{S}^{N}$, for $i=1,2,3$, such that

$$
\left(-b_{i},-\Phi_{i},-X_{i}\right) \in \bar{P}^{2,-} v_{i}, \quad i=1,2, \quad\left(\frac{b_{3}}{2}, \frac{\Phi_{3}}{2}, \frac{1}{2} X_{3}\right) \in \bar{P}^{2,+} v_{3},
$$

and if $O$ denotes the null $N \times N$ matrix, we have

$$
\begin{aligned}
\left(\begin{array}{ccc}
X_{1} & 0 & 0 \\
0 & X_{2} & 0 \\
0 & 0 & X_{3}
\end{array}\right) & \leq \nabla^{2} \Phi(\widehat{\xi}, \widehat{t})+\kappa\left[\nabla^{2} \Phi(\widehat{\xi}, \widehat{t})\right]^{2} \\
b_{1}+b_{2}+b_{3} & =\partial_{t} \Phi(\widehat{\xi}, \widehat{t}) .
\end{aligned}
$$

Where for the sake of simplifying the notation, we have set $v_{1}, v_{2}, v_{3}$ for $v(\widehat{x}, \widehat{t}), v(\widehat{y}, \widehat{t})$, $v(\widehat{z}, \widehat{t})$, respectively, and in the same way $\Phi_{1}, \Phi_{2}, \Phi_{3}$, for the partial derivatives of $\Phi$ evaluated at the maximum point. Now we compute the derivatives of $\Phi$. Set

$$
p=\frac{2 M}{\delta}(\widehat{x}-\widehat{z})|\widehat{x}-\widehat{z}|^{2}, \quad q=\frac{2 M}{\delta}(\widehat{y}-\widehat{z})|\widehat{y}-\widehat{z}|^{2}, \quad m=\frac{2 M}{\delta}(\widehat{x}+\widehat{y}-2 \widehat{z})
$$

Then

$$
\partial_{t} \Phi(\widehat{\xi}, \widehat{t})=\frac{\gamma}{(T-\widehat{t})^{2}} \geq \frac{\gamma}{T^{2}}, \quad \text { and } \quad\left\{\begin{array}{l}
\Phi_{1}=2 \varepsilon \widehat{x}+2 p+m \\
\Phi_{2}=2 \varepsilon \widehat{y}+2 q+m \\
\Phi_{3}=2 \varepsilon \widehat{z}-2 p-2 q-2 m
\end{array}\right.
$$

Moreover, let $I, I_{3 N}$ be the identity matrices respectively of order $N$ and $3 N$, then define

$$
A=\left(\begin{array}{ccc}
|p|^{\frac{2}{3}} I & 0 & -|p|^{\frac{2}{3}} I \\
0 & |q|^{\frac{2}{3}} I & -|q|^{\frac{2}{3}} I \\
-|p|^{\frac{2}{3}} I & -|q|^{\frac{2}{3}} I & \left(|p|^{\frac{2}{3}}+|q|^{\frac{2}{3}}\right) I
\end{array}\right), B=\left(\begin{array}{ccc}
\frac{p \otimes p}{|p|^{\frac{4}{3}}} & 0 & -\frac{p \otimes p}{|p|^{\frac{4}{3}}} \\
0 & \frac{q \otimes q}{|q|^{\frac{4}{3}}} & -\frac{q \otimes q}{|q|^{\frac{4}{3}}} \\
-\frac{p \otimes p}{|p|^{\frac{4}{3}}} & -\frac{q \otimes q}{|q|^{\frac{4}{3}}} & \frac{p \otimes p}{|p|^{\frac{4}{3}}}+\frac{q \otimes q}{|q|^{\frac{4}{3}}}
\end{array}\right)
$$

thus it holds that

$$
\nabla^{2} \Phi(\widehat{\xi}, \widehat{t})=\left(\frac{2 M}{\delta}\right)^{\frac{1}{3}}\{2 A+4 B\}+\frac{2 M}{\delta}\left(\begin{array}{ccc}
I & I & -2 I \\
I & I & -2 I \\
-2 I & -2 I & 4 I
\end{array}\right)+2 \varepsilon I_{3 N}
$$


By (4.11) and equation (4.3), we have

$$
\begin{aligned}
b_{i} \leq & \frac{1}{2} \operatorname{tr}\left(\sigma \sigma^{\top} X_{i}\right)-\left\langle\mu_{i}, \Phi_{i}\right\rangle+\lambda\left(v_{i} e^{C \widehat{t}}\right) e^{C \widehat{t}}\left|\sigma^{\top} \Phi_{i}\right|^{2} \\
& -\eta\left(v_{i} e^{C \widehat{t}}\right)\left\langle\sigma^{\top} \Phi_{i}, w_{i}\right\rangle+C v_{i}+e^{-C \widehat{t}} f_{i}, \quad i=1,2 \\
b_{3} \leq & \frac{1}{2} \operatorname{tr}\left(\sigma \sigma^{\top} X_{3}\right)-\left\langle\mu_{3}, \Phi_{3}\right\rangle-2 \lambda\left(v_{3} e^{C \widehat{t}}\right) e^{C \widehat{t}}\left|\frac{1}{2} \sigma^{\top} \Phi_{3}\right|^{2} \\
& -\eta\left(v_{3} e^{C \widehat{t}}\right)\left\langle\sigma^{\top} \Phi_{3}, w_{3}\right\rangle-2 e^{-C \widehat{t}} f_{3}-2 C v_{3} .
\end{aligned}
$$

Here we used the same notation of (4.11) to denote the functions $\mu, w, f$, and we have also omitted the dependence of the matrix $\sigma$ on $\widehat{t}$. Adding inequalities (4.18) and using (3.25), we get

$$
\begin{aligned}
& \partial_{t} \Phi(\widehat{\xi}, \widehat{t}) \leq \frac{1}{2} \operatorname{tr}\left(\sigma \sigma^{\top}\left(X_{1}+X_{2}+X_{3}\right)\right)-\left[\left\langle\mu_{1}, \Phi_{1}\right\rangle+\left\langle\mu_{2}, \Phi_{2}\right\rangle+\left\langle\mu_{3}, \Phi_{3}\right\rangle\right] \\
& +\left[\lambda\left(v_{1} e^{C \widehat{t}}\right) e^{C \widehat{t}}\left|\sigma^{\top} \Phi_{1}\right|^{2}-\eta\left(v_{1} e^{C \widehat{t}}\right)\left\langle\sigma^{\top} \Phi_{1}, w_{1}\right\rangle+\lambda\left(v_{2} e^{C \widehat{t}}\right) e^{C \widehat{t}}\left|\sigma^{\top} \Phi_{2}\right|^{2}\right. \\
& \left.-\eta\left(v_{2} e^{C \widehat{t}}\right)\left\langle\sigma^{\top} \Phi_{2}, w_{2}\right\rangle-2 \lambda\left(v_{3} e^{C \widehat{t}}\right) e^{C \widehat{t}}\left|\frac{1}{2} \sigma^{\top} \Phi_{3}\right|^{2}-\eta\left(v_{3} e^{C \widehat{t}}\right)\left\langle\sigma^{\top} \Phi_{3}, w_{3}\right\rangle\right] \\
& +C\left(v_{1}+v_{2}-2 v_{3}\right)+e^{-C \widehat{t}}\left[f_{1}+f_{2}-2 f_{3}\right] .
\end{aligned}
$$

Now we use (4.15) and (4.17) for estimating the single part in the brackets of the inequality (4.19). Let $\sigma^{(l)}$ be the $l$ column of $\sigma$, and for $l=1, \ldots, d$, let $\Sigma^{(l)}$ be the $3 N$-dimensional vector whose transpose is $\left(\left(\sigma^{(l)}\right)^{\top},\left(\sigma^{(l)}\right)^{\top},\left(\sigma^{(l)}\right)^{\top}\right)$, then by $(4.12)$, we infer

$$
\begin{gathered}
\operatorname{tr}\left(\sigma \sigma^{\top}\left(X_{1}+X_{2}+X_{3}\right)\right)=\sum_{l=1}^{d}\left(\left\langle X_{1} \sigma^{(l)}, \sigma^{(l)}\right\rangle+\left\langle X_{2} \sigma^{(l)}, \sigma^{(l)}\right\rangle+\left\langle X_{3} \sigma^{(l)}, \sigma^{(l)}\right\rangle\right) \\
\leq \sum_{l=1}^{d}\left(\left\langle\nabla^{2} \Phi(\widehat{\xi}, \widehat{t}) \Sigma^{(l)}, \Sigma^{(l)}\right\rangle+\kappa\left|\nabla^{2} \Phi(\widehat{\xi}, \widehat{t}) \Sigma^{(l)}\right|^{2}\right)
\end{gathered}
$$

Using (4.17), it is easy to compute that $\nabla^{2} \Phi(\widehat{\xi}, \widehat{t}) \Sigma^{(l)}=2 \varepsilon \Sigma^{(l)}$. So we deduce that

$$
\operatorname{tr}\left(\sigma \sigma^{\top}\left(X_{1}+X_{2}+X_{3}\right)\right) \leq 18 \varepsilon \sum_{l=1}^{d}\left|\sigma^{(l)}\right|^{2}=O(\varepsilon) .
$$

By condition (4.8), we have

$$
\begin{aligned}
& \varepsilon|\widehat{x}|, \varepsilon|\widehat{y}|, \varepsilon|\widehat{z}| \leq O(\sqrt{\varepsilon}), \\
& \frac{1}{2}\left[\left(\frac{\delta}{2 M}\right)^{\frac{1}{3}}\left(|p|^{\frac{4}{3}}+|q|^{\frac{4}{3}}\right)+\frac{\delta}{2 M}|m|^{2}\right]+M \delta \leq 2 v_{3}-v_{1}-v_{2} .
\end{aligned}
$$

Therefore, using the notations introduced in (4.14)-(4.15) and (4.22) and the regularity 
assumptions on $\mu$, we obtain.

$$
\begin{aligned}
& \left|\left\langle\mu_{1}, \Phi_{1}\right\rangle+\left\langle\mu_{2}, \Phi_{2}\right\rangle+\left\langle\mu_{3}, \Phi_{3}\right\rangle\right| \leq \\
& \leq\left|\left\langle\mu_{1}, 2 p+m\right\rangle+\left\langle\mu_{2}, 2 q+m\right\rangle-\left\langle\mu_{3}, 2 p+2 q+2 m\right\rangle\right|+2 \varepsilon\left|\left\langle\mu_{1}, \widehat{x}\right\rangle+\left\langle\mu_{2}, \widehat{y}\right\rangle+\left\langle\mu_{3}, \widehat{z}\right\rangle\right| \\
& \leq\left|2\left\langle\mu_{1}-\mu_{3}, p\right\rangle+2\left\langle\mu_{2}-\mu_{3}, q\right\rangle+\left\langle\mu_{1}+\mu_{2}-2 \mu_{3}, m\right\rangle\right|+O(\sqrt{\varepsilon}) \\
& \leq O(\sqrt{\varepsilon})+\sup _{t \in(0, T)}\|\mu(t)\|_{\mathbb{W}^{2}, \infty} \times \\
& \quad\left[2\left(\frac{\delta}{2 M}\right)^{\frac{1}{3}}\left(|p|^{\frac{4}{3}}+|q|^{\frac{4}{3}}\right)+\left[\left(\frac{\delta}{2 M}\right)^{\frac{1}{3}}\left(|p|^{\frac{4}{3}}+|q|^{\frac{4}{3}}\right)+\frac{\delta}{2 M}|m|^{2}\right]^{\frac{1}{2}}\left(\sqrt{\frac{\delta}{2 M}}|m|\right)\right] \\
& \leq \sup _{t \in(0, T)}\|\mu(t)\|_{\mathbb{W} 2, \infty}\left[4\left(2 v_{3}-v_{1}-v_{2}\right)+\frac{1}{2}\left[\left(\frac{\delta}{2 M}\right)^{\frac{1}{3}}\left(|p|^{\frac{4}{3}}+|q|^{\frac{4}{3}}\right)+\frac{\delta}{2 M}|m|^{2}\right]\right. \\
& \left.\quad+\frac{\delta}{4 M}|m|^{2}\right]+O(\sqrt{\varepsilon}) \leq 6 \sup _{t \in(0, T)}\|\mu(t)\|_{\mathbb{W} 2, \infty}\left(2 v_{3}-v_{1}-v_{2}\right)+O(\sqrt{\varepsilon}),
\end{aligned}
$$

where in the last inequality we have again used (4.22). For estimating the nonlinear part we consider the function

$$
G(u, \theta)=\lambda(u) e^{C \widehat{t}}\left|\sigma^{\top} \theta\right|^{2}-\eta(u)\left\langle\sigma^{\top} \theta, w_{3}\right\rangle
$$

which depends on $(u, \theta) \in I \times \mathbb{R}^{N}$, and for every $u_{1}, u_{2}, \theta_{1}, \theta_{2}$, define

$$
\Delta^{2} G\left(u_{1}, u_{2}, \theta_{1}, \theta_{2}\right)=G\left(u_{1}, \theta_{1}\right)+G\left(u_{2}, \theta_{2}\right)-2 G\left(\frac{u_{1}+u_{2}}{2}, \frac{\theta_{1}+\theta_{2}}{2}\right) .
$$

Let $\eta_{i}$ denote the value of $\eta$ at $v_{i} e^{C \widehat{t}}$, for $i=1,2,3$, then using notations (4.24), (4.25) and (4.14)-(4.15), we get

$$
\begin{aligned}
& {\left[\lambda\left(v_{1} e^{C \widehat{t}}\right) e^{C \widehat{t}}\left|\sigma^{\top} \Phi_{1}\right|^{2}-\eta\left(v_{1} e^{C \widehat{t}}\right)\left\langle\sigma^{\top} \Phi_{1}, w_{1}\right\rangle+\lambda\left(v_{2} e^{C \widehat{t}}\right) e^{C \widehat{t}}\left|\sigma^{\top} \Phi_{2}\right|^{2}\right.} \\
& \left.\quad-\eta\left(v_{2} e^{C \widehat{t}}\right)\left\langle\sigma^{\top} \Phi_{2}, w_{2}\right\rangle-2 \lambda\left(v_{3} e^{C \widehat{t}}\right) e^{C \widehat{t}}\left|\frac{1}{2} \sigma^{\top} \Phi_{3}\right|^{2}-\eta\left(v_{3} e^{C \widehat{t}}\right)\left\langle\sigma^{\top} \Phi_{3}, w_{3}\right\rangle\right]= \\
& =\Delta^{2} G\left(v_{1} e^{C \widehat{t}}, v_{2} e^{C \widehat{t}}, 2 p+m, 2 q+m\right)+2\left[G\left(\frac{\left(v_{1}+v_{2}\right) e^{C \widehat{t}}}{2}, p+q+m\right)-\right. \\
& \left.\quad-G\left(v_{3} e^{C \widehat{t}}, p+q+m\right)\right]+\left[\eta_{1}\left\langle\sigma^{\top}(2 p+m), w_{3}-w_{1}\right\rangle+\right. \\
& \left.\quad+\eta_{2}\left\langle\sigma^{\top}(2 q+m), w_{3}-w_{2}\right\rangle\right]+O(\sqrt{\varepsilon}) .
\end{aligned}
$$

In the last passages we used the inequalities (4.22) for estimating the residual terms which involve $\varepsilon$.

$$
\begin{gathered}
\eta_{1}\left\langle\sigma^{\top}(2 p+m), w_{3}-w_{1}\right\rangle+\eta_{2}\left\langle\sigma^{\top}(2 q+m), w_{3}-w_{2}\right\rangle= \\
=\left[\eta_{1}-\eta_{3}\right]\left\langle\sigma^{\top}(2 p+m), w_{3}-w_{1}\right\rangle+\left[\eta_{2}-\eta_{3}\right]\left\langle\sigma^{\top}(2 q+m), w_{3}-w_{2}\right\rangle \\
+2 \eta_{3}\left[\left\langle\sigma^{\top} p, w_{3}-w_{1}\right\rangle+\left\langle\sigma^{\top} q, w_{3}-w_{2}\right\rangle\right]-\eta_{3}\left\langle\sigma^{\top} m, w_{1}+w_{2}-2 w_{3}\right\rangle .
\end{gathered}
$$

Hence, using the Lipschitz regularity of the function $u$ and the regularity of $w$, jointly 
with (4.22), we deduce.

$$
\begin{aligned}
& {\left[\eta_{1}-\eta_{3}\right]\left\langle\sigma^{\top}(2 p+m), w_{3}-w_{1}\right\rangle \leq\left\|\eta^{\prime}\right\|_{\infty}\left\|\sigma^{\top}\right\|_{\infty} \sup _{t \in(0, T)}\|w(t)\|_{\mathbb{W} 2, \infty}\left[4\|u\|_{\infty}\left(\frac{\delta}{2 M}\right)^{\frac{1}{3}}|p|^{\frac{4}{3}}\right.} \\
& \left.+\sup _{t \in[0, T)}\|u(\cdot, t)\|_{\mathbb{W} 1, \infty}\left(\frac{\delta}{2 M}\right)^{\frac{2}{3}}|p|^{\frac{2}{3}}|m|\right] \leq \\
& \quad \leq\left\|\eta^{\prime}\right\|_{\infty}\left\|\sigma^{\top}\right\|_{\infty} \sup _{t \in(0, T)}\|w(t)\|_{\mathbb{W}^{2}, \infty}\left[4\|u\|_{\infty}\left(\frac{\delta}{2 M}\right)^{\frac{1}{3}}|p|^{\frac{4}{3}}+\frac{1}{2} \sup _{t \in[0, T)}\|u(t)\|_{\mathbb{W} 1, \infty}\right. \\
& \left.\left.\quad\left(\left(\frac{\delta}{2 M}\right)^{\frac{1}{3}}|p|^{\frac{4}{3}}+\frac{\delta}{2 M}|m|^{2}\right)\right] \leq C_{1}\left[\frac{1}{2}\left(\frac{\delta}{2 M}\right)^{\frac{1}{3}}|p|^{\frac{4}{3}}+\frac{1}{4} \frac{\delta}{2 M}|m|^{2}\right)\right] .
\end{aligned}
$$

In the last inequality, we introduced the notation

$$
C_{1}=2\left\|\eta^{\prime}\right\|_{\infty}\left\|\sigma^{\top}\right\|_{\infty} \sup _{t \in(0, T)}\|w(t)\|_{\mathbb{W}^{2}, \infty} \max \left(8\|u\|_{\infty}, \sup _{t \in[0, T)}\|u(t)\|_{\mathbb{W}^{1}, \infty}\right)
$$

We can repeat the argument for estimating $\left[\eta_{2}-\eta_{3}\right]\left\langle\sigma^{\top}(2 q+m), w_{3}-w_{2}\right\rangle$, again using the second relation in (4.22). We obtain the inequality

$$
\begin{array}{r}
{\left[\eta_{1}-\eta_{3}\right]\left\langle\sigma^{\top}(2 p+m), w_{3}-w_{1}\right\rangle+\left[\eta_{2}-\eta_{3}\right]\left\langle\sigma^{\top}(2 q+m), w_{3}-w_{2}\right\rangle \leq} \\
\left.\leq C_{1}\left[\frac{1}{2}\left(\frac{\delta}{2 M}\right)^{\frac{1}{3}}\left(|p|^{\frac{4}{3}}+|q|^{\frac{4}{3}}\right)+\frac{1}{2} \frac{\delta}{2 M}|m|^{2}\right)\right] \leq C_{1}\left(2 v_{3}-v_{1}-v_{2}\right)
\end{array}
$$

By (4.22) and the following inequality,

$$
\left|w_{1}+w_{2}-2 w_{3}\right| \leq \sup _{t \in(0, T)}\|w(t)\|_{\mathbb{W}^{2}, \infty}\left[\left(\frac{\delta|p|}{2 M}\right)^{\frac{4}{3}}+\left(\frac{\delta|q|}{2 M}\right)^{\frac{4}{3}}+\left(\frac{\delta|m|}{2 M}\right)^{2}\right]^{\frac{1}{2}},
$$

it is easy to estimate the last two terms in the brackets [.] in (4.27) through the expression $C_{2}\left(2 v_{3}-v_{1}-v_{2}\right)$, where

$$
C_{2}=5\|\eta\|_{\infty}\left\|\sigma^{\top}\right\|_{\infty} \sup _{t \in(0, T)}\|w(t)\|_{\mathbb{W}^{2}, \infty}
$$

Now we proceed with the estimates for the first two terms in (4.26). We observe that by the assumption $i i$ ), we can consider the positive constant,

$$
C_{3}=\frac{\left\|\eta^{\prime}\right\|_{\infty}^{2}\|w\|_{\infty}^{2}}{4 \min _{I} \lambda^{\prime}}
$$

and we can also write

$$
\partial_{u} G(u, \theta) \geq-C_{3} e^{-C \widehat{t}}, \quad \forall(u, \theta) \in[a, b] \times \mathbb{R}^{N} .
$$

Therefore by (4.33) and $2 v_{3}-v_{1}-v_{2}>0$, the following holds:

$$
G\left(\frac{\left(v_{1}+v_{2}\right) e^{C \widehat{t}}}{2}, p+q+m\right)-G\left(v_{3} e^{C \hat{t}}, p+q+m\right) \leq \frac{1}{2} C_{3}\left(2 v_{3}-v_{1}-v_{2}\right) .
$$

Assumption iii) yields $w_{3}=\sigma^{\top} b_{3}$, for some $N$-dimensional vector $b_{3}$. Moreover,

$$
\partial_{u}^{2} G=e^{C \widehat{t}} \lambda^{\prime \prime}\left|\sigma^{\top} \theta\right|^{2}-\eta^{\prime \prime}\left\langle\sigma^{\top} \theta, w_{3}\right\rangle, \quad \partial_{\theta, u}^{2} G=\sigma \sigma^{\top} J, \quad \partial_{\theta}^{2} G=2 e^{C \widehat{t}} \lambda \sigma \sigma^{\top},(
$$


where $J=2 e^{C \widehat{t}} \lambda^{\prime} \theta-\eta^{\prime} b_{3}$. Let $X=(k, h) \in \mathbb{R} \times \mathbb{R}^{N}$, and consider the orthogonal matrix $A$ such that $A \sigma \sigma^{\top} A^{\top}$ is diagonal, with entries $S_{i} \geq 0, i=1, \ldots, N$, and set $\bar{h}=A h, \bar{J}=A J$. Define

$$
C_{4}=\frac{\left\|\eta^{\prime \prime}-2\left(\lambda^{\prime} / \lambda\right) \eta^{\prime}\right\|_{\infty}^{2}\|w\|_{\infty}^{2}}{4 \min _{I}\left(2 \frac{\left(\lambda^{\prime}\right)^{2}}{\lambda}-\lambda^{\prime \prime}\right)}-\frac{1}{2 \max _{I}(\lambda)}\left\|\eta^{\prime}\right\|_{\infty}^{2}\|w\|_{\infty}^{2}
$$

By (4.35), ii) and (4.36), we have

$$
\begin{aligned}
& \left\langle\left(\nabla^{2} G-C_{4} e^{-C \widehat{t}}\left(\begin{array}{ll}
I & 0 \\
0 & 0
\end{array}\right)\right) X, X\right\rangle=\left(\partial_{u}^{2} G-C_{4} e^{-C \widehat{t}}\right) k^{2}+\sum_{i=1}^{N}\left(2 e^{C \widehat{t}} \lambda \bar{h}_{i}^{2}+2 k \bar{J}_{i} \bar{h}_{i}\right) S_{i} \\
\leq & {\left[\partial_{u}^{2} G-C_{4} e^{-C \widehat{t}}-\sum_{i=1}^{N} \frac{e^{-C \widehat{t}}}{2 \lambda} S_{i}\left|\bar{J}_{i}\right|^{2}\right] k^{2} } \\
= & {\left[e^{C \widehat{t}} \lambda^{\prime \prime}\left|\sigma^{\top} \theta\right|^{2}-\eta^{\prime \prime}\left\langle\sigma^{\top} \theta, w_{3}\right\rangle-C_{4} e^{-C \widehat{t}}-\frac{e^{-C \widehat{t}}}{2 \lambda}\left|2 e^{C \widehat{t}} \lambda^{\prime} \sigma^{\top} \theta-\eta^{\prime} w_{3}\right|^{2}\right] k^{2} } \\
= & {\left[e^{C \widehat{t}}\left(\lambda^{\prime \prime}-2 \frac{\left(\lambda^{\prime}\right)^{2}}{\lambda}\right)\left|\sigma^{\top} \theta\right|^{2}-\left[\eta^{\prime \prime}-2\left(\lambda^{\prime} / \lambda\right) \eta^{\prime}\right]\left\langle\sigma^{\top} \theta, w_{3}\right\rangle-\frac{e^{-C \widehat{t}}}{2 \lambda}\left|\eta^{\prime}\right|^{2}\left|w_{3}\right|^{2}-C_{4} e^{-C \widehat{t}}\right] k^{2} } \\
\leq & {\left[e^{-C \widehat{t}} \frac{\left\|\eta^{\prime \prime}-2\left(\lambda^{\prime} / \lambda\right) \eta^{\prime}\right\|_{\infty}^{2}\|w\|_{\infty}^{2}}{4 \min _{I}\left(2 \frac{\left(\lambda^{\prime}\right)^{2}}{\lambda}-\lambda^{\prime \prime}\right)}-\frac{e^{-C \widehat{t}}}{2 \max _{I}(\lambda)}\left\|\eta^{\prime}\right\|_{\infty}^{2}\|w\|_{\infty}^{2}-C_{4} e^{-C \widehat{t}}\right] k^{2}=0 . }
\end{aligned}
$$

Set the constant

$$
C_{5}=\frac{1}{2} C_{4}\left(1+\left[\frac{1}{2 M^{2}}-1\right]_{+}\right) \sup _{t \in[0, T)}\|u(t)\|_{\mathbb{W}^{1, \infty}}^{2},
$$

where $[\cdot]_{+}$denotes the positive part of a real number; therefore (4.37), (4.14)-(4.15), (4.22) and the Lipschitz regularity of $u$ imply

$$
\begin{aligned}
& \Delta^{2} G\left(v_{1} e^{C \widehat{t}}, v_{2} e^{C \widehat{t}}, 2 p+m, 2 q+m\right)=\Delta^{2}\left(G-C_{4} e^{-C \widehat{t}} \frac{u^{2}}{2}\right)+\frac{1}{4} C_{4} e^{C \widehat{t}}\left|v_{1}-v_{2}\right|^{2} \\
& \leq \frac{1}{4} C_{4} e^{C \widehat{t}}\left|v_{1}-v_{2}\right|^{2} \leq \frac{1}{2} C_{4}\left(\left|u_{1}-u_{3}\right|^{2}+\left|u_{2}-u_{3}\right|^{2}\right) \leq \frac{1}{2} C_{4} \sup _{t \in[0, T)}\|u(t)\|_{\mathbb{W} 1, \infty}^{2} \times \\
& {\left[\left(\frac{\delta|p|}{2 M}\right)^{\frac{2}{3}}+\left(\frac{\delta|q|}{2 M}\right)^{\frac{2}{3}}\right] \leq \frac{1}{2} C_{4} \sup _{t \in[0, T)}\|u(t)\|_{\mathbb{W}^{1}, \infty}^{2}\left(\frac{1}{2}\left[\left(\frac{\delta}{2 M}\right)^{\frac{1}{3}}\left(|p|^{\frac{4}{3}}+|q|^{\frac{4}{3}}\right)\right]+\frac{\delta}{2 M}\right)} \\
& \leq \frac{1}{2} C_{4} \sup _{t \in[0, T)}\|u(t)\|_{\mathbb{W} 1, \infty}^{2}\left(\left(2 v_{3}-v_{1}-v_{2}\right)+\delta M\left[\frac{1}{2 M^{2}}-1\right]_{+}\right) \leq C_{5}\left(2 v_{3}-v_{1}-v_{2}\right) .
\end{aligned}
$$

Introducing the estimates (4.30), (4.34) and (4.39). in (4.26), we finally obtain

$$
\begin{aligned}
& {\left[\lambda\left(v_{1} e^{C \widehat{t}}\right) e^{C \widehat{t}}\left|\sigma^{\top} \Phi_{1}\right|^{2}-\eta\left(v_{1} e^{C \widehat{t}}\right)\left\langle\sigma^{\top} \Phi_{1}, w_{1}\right\rangle+\lambda\left(v_{2} e^{C \widehat{t}}\right) e^{C \widehat{t}}\left|\sigma^{\top} \Phi_{2}\right|^{2}\right.} \\
& \left.-\eta\left(v_{2} e^{C \widehat{t}}\right)\left\langle\sigma^{\top} \Phi_{2}, w_{2}\right\rangle-2 \lambda\left(v_{3} e^{C \widehat{t}}\right) e^{C \widehat{t}}\left|\frac{1}{2} \sigma^{\top} \Phi_{3}\right|^{2}-\eta\left(v_{3} e^{C \widehat{t}}\right)\left\langle\sigma^{\top} \Phi_{3}, w_{3}\right\rangle\right] \leq \\
& \leq\left(C_{1}+C_{2}+C_{3}+C_{5}\right)\left(2 v_{3}-v_{1}-v_{2}\right)+O(\sqrt{\varepsilon}) .
\end{aligned}
$$

Consider now the last term in (4.19). By the regularity assumptions on the function 
$f$, the same argument used to estimate $\left|u_{1}-u_{2}\right|$ and again by (4.22), we have

$$
\begin{aligned}
& f_{1}+f_{2}-2 f_{3} \leq \sup _{t \in(0, T)}\|f(t)\|_{\mathbb{W}^{2}, \infty}\left[\left[\left(\frac{\delta}{2 M}\right)^{\frac{4}{3}}\left(|p|^{\frac{4}{3}}+|q|^{\frac{4}{3}}\right)+\left(\frac{\delta}{2 M}\right)^{2}|m|^{2}\right]^{\frac{1}{2}}\right. \\
& \left.+\left|u_{1}-u_{3}\right|^{2}+\left|u_{2}-u_{3}\right|^{2}+\left|u_{1}+u_{2}-2 u_{3}\right|\right] \leq \sup _{t \in(0, T)}\|f(t)\|_{\mathbb{W}^{2}, \infty}\left\{2 v_{3}-v_{1}-v_{2}\right. \\
& \left.+\delta M\left[\frac{1}{4 M^{2}}-1\right]_{+}+2\left(\frac{C_{5}}{C_{4}}\right)\left(2 v_{3}-v_{1}-v_{2}\right)+e^{C \widehat{t}}\left(2 v_{3}-v_{1}-v_{2}\right)\right\} \\
& \quad \leq C_{6}\left(2 v_{3}-v_{1}-v_{3}\right),
\end{aligned}
$$

where

$$
C_{6}=\sup _{t \in(0, T)}\|f(t)\|_{\mathbb{W}^{2}, \infty}\left(1+\left[\frac{1}{4 M^{2}}-1\right]_{+}+2\left(\frac{C_{5}}{C_{4}}\right)+e^{C \widehat{t}}\right) .
$$

Finally, using (4.21), (4.23), (4.40), and (4.41) in (4.19), we get

$$
\frac{\gamma}{T^{2}} \leq\left[6 \sup _{t \in(0, T)}\|\mu(t)\|_{\mathbb{W} 2, \infty}+\sum_{i=1}^{5} C_{i}+C_{6} e^{-C \widehat{t}}-C\right]\left(2 v_{3}-v_{1}-v_{3}\right)+O(\sqrt{\varepsilon}) \cdot(
$$

From the definition (4.42), we see that $C_{6} e^{-C \widehat{t}}$ is bounded as a function of $C>0$, so we can choose $C$ to be sufficently large to obtain a contradiction letting $\varepsilon \rightarrow 0$. This proves the result.

Now we use these Propositions to eliminate the conditions on $\lambda$ and to obtain the assertion of Theorems 3.2 and 3.3.

Proof of Theorem 3.2. We must now build a change of variable such that the new differential equation will have the required structural properties of Proposition 4.1.

Let $c, \Lambda$ be respectively the infimum of the closed interval $I$, where $u$ takes its values, and the primitive of $\lambda$ with $\Lambda(c)=0$. Then, consider the solution $Q=Q(\tau)$ of the following ordinary Cauchy problem:

$$
\left\{\begin{array}{l}
\frac{d Q}{d \tau}=\exp (4 \sqrt{\tau+1}+2 \Lambda(Q)) \\
Q(0)=c
\end{array}\right.
$$

The problem admits an increasing local solution. Moreover by the continuity of $\lambda, Q$ is also $\mathcal{C}^{2}$. Let $\bar{Q}$ be the supremum of $Q$, then we show that $\bar{Q} \geq b$. Consider the following cases:

* Case $1,(b=\infty)$. Let $\left[0, \tau^{\star}\right)$ be the maximal interval of existence for $Q$. Then $\bar{Q}$ is the limit of $Q$ for $\tau \rightarrow \tau^{*}$. If $\tau^{\star}=\infty$, then $\bar{Q}=\infty$. If this is not the case (i.e. $\bar{Q}<\infty)$, then by the equation (4.44),

$$
Q^{\prime}(\tau) \geq \exp \left(2 \inf _{q \in[c, \bar{Q}]} \Lambda(q)+4\right), \quad \forall \tau>0 .
$$

So by integrating (4.45) from 0 to $\tau>0$ and letting $\tau$ go to infinity we obtain a contradiction. If $\tau^{\star}<\infty$, then by definition of maximal interval, this results in a blow up of $Q$ at $\tau^{\star}$. Otherwise, since $\lambda$ is defined in $(a, \infty)$, the solution $Q$ could be extended.

* Case 2, $(b<\infty)$. Consider again the maximal interval of existence. If $\tau^{\star}=\infty$ and $\bar{Q}$ is strictly less than $b$, then, again using (4.45), we obtain the same contradition as 
before.

If $\tau^{\star}<\infty$ and $\bar{Q}<b$, then the solution $Q$ can be extended because $\lambda$ is continuous in $(\bar{Q}, b)$. In each case, the function reaches $b$ in the limit sense; in particular, $Q$ maps $I$. Moreover, $Q$ can be defined in an open interval $V \subset\left(-\varepsilon_{0}, \infty\right), \varepsilon_{0}>0$, with $I \subset Q(V) \subset(a, b)$. Since $\mathrm{Q}$ is a strictly increasing function, it admits a $\mathcal{C}^{2}(Q(V) ; V)$ inverse denoted by $P$. We use $P$ as a transformation for a global change of the variable $u$. The function $\tau=P \circ u$, is a bounded, $t$-uniformly Lipschitz continuous viscosity solution of

$$
\begin{aligned}
\partial_{t} \tau-\frac{1}{2} \operatorname{tr}\left(\sigma \sigma^{\top} \nabla^{2} \tau\right)+\langle\mu, \nabla \tau\rangle-\frac{\left|\sigma^{\top} \nabla \tau\right|^{2}}{\sqrt{\tau+1}}+ \\
+\eta(Q(\tau))\left\langle\sigma^{\top} \nabla \tau, w\right\rangle+f(x, t, Q(\tau))=0,
\end{aligned}
$$

in $\mathbb{R}^{N} \times(0, T)$, where the initial datum is $\tau_{0}=P \circ u_{0}$, which takes values in the closed interval $P(I)$. It is easy to verify the structural hypotheses $i$ ), $i i$ ) of Proposition 4.1, where $\lambda$ and $\eta$ are substituted, respectively, by the functions $-(1+\tau)^{-\frac{1}{2}}$ and $\eta \circ Q$, over the interval $V$, with regularity assumptions over $P(I)$. So applying Proposition 4.1, we deduce that there exist positive constants $C, K_{0}>0$, such that

$$
\tau(x+h, t)+\tau(x-h, t)-2 \tau(x, t) \geq-e^{C t} K_{0}|h|^{2}, \forall x, h \in \mathbb{R}^{N}, t \in[0, T),
$$

where the constant $K_{0}$ depends on $P, L_{0}$ and on $\operatorname{Lip}\left(u_{0}\right)$. Therefore for every $x, h \in$ $\mathbb{R}^{N}, t \in[0, T)$, and $s^{+}, s^{-} \in[0,1]$, we define

$$
\tau=\tau(x, t), \quad \tau^{+}=\tau+s^{+}(\tau(x+h, t)-\tau), \quad \tau^{-}=\tau+s^{-}(\tau(x-h, t)-\tau)
$$

For $g=u$ and $g=\tau$, let $\Delta_{h}^{2} g(x, t)=g(x+h, t)+g(x-h, t)-2 g(x, t)$. Then for some $s^{+}, s^{-}$, we can write,

$$
\begin{aligned}
& \Delta_{h}^{2} u(x, t)=Q^{\prime}\left(\tau^{+}\right)(\tau(x+h, t)-\tau)+Q^{\prime}\left(\tau^{-}\right)(\tau(x-h, t)-\tau)= \\
& =Q^{\prime}(\tau) \Delta_{h}^{2} h(x, t)+s^{+} Q^{\prime \prime}\left(\tau^{++}\right)(\tau(x+h, t)-\tau)^{2}+s^{-} Q^{\prime \prime}\left(\tau^{--}\right)(\tau(x-h, t)-\tau)^{2} \\
& \geq-\left[K_{0} C_{00} e^{C t}+c_{0}\right]|h|^{2} .
\end{aligned}
$$

for some $\tau^{++} \in\left[\min \left(\tau, \tau^{+}\right), \max \left(\tau, \tau^{+}\right)\right], \tau^{--} \in\left[\min \left(\tau, \tau^{-}\right), \max \left(\tau, \tau^{-}\right)\right]$, where $C_{00}$ is a positive constant depending on $Q^{\prime}$, while $c_{0}$ depends on the Lipschitz constant of the solution $u$ and on $Q^{\prime \prime}$. This proves Theorem 3.2.

The equivalent result for the semiconcavity property can be obtained with the same arguments. So we limit ourselves to giving some outlines in the following proof.

Proof of Theorem 3.3. By the same notations used for proving Theorem 3.2, we choose the increasing transformation $u=Q(\tau)$, where $Q$ is implicitly defined as the solution of the ordinary Cauchy problem,

$$
\left\{\begin{aligned}
\frac{d Q}{d \tau} & =\exp \left(-\frac{2}{l+1}(\tau+1)^{l+1}+2 \Lambda(Q)\right) \\
Q(0) & =c
\end{aligned}\right.
$$

Here $l$ is chosen to be bigger than 3. As in the semiconvexity case, a new equation for $\tau$, which satisfies the structural hypothesis required for applying Proposition 4.2, is obtained.

Theorem 2.4 is an immediate consequence of Theorems 3.2 and 3.3. This conclusive fact allows us to get a second-order regularity result for the solution of problem (1.1), with regular initial data. 


\section{Time regularity}

Using the spatial regularity of the solution $u$, given in Theorem 3.4, we are able to derive the time regularity. That regularity has not been stated for second order Hamilton-Jacoby equations which have the structure of (1.1). The following result is based on the application of the comparison principle.

TheOREM 5.1. Let $u(t) \in \mathbb{W}^{2, \infty}\left(\mathbb{R}^{N}\right)$, uniformly bounded for $t \in[0, T)$. Assume that $\sigma, \mu, w, f$ and $\lambda, \eta$ are continuous and bounded functions of their variables, then $u(x, \cdot)$ is a Lipschitz continuous function uniformly in $x \in \mathbb{R}^{N}$.

Proof of Theorem 5.1. By the assumption on $u, u$ and $\nabla u$ are bounded, therefore $u$ is a viscosity subsolution of

$$
\partial_{t} u-\frac{1}{2} \operatorname{tr}\left(\sigma(t) \sigma(t)^{\top} \nabla^{2} u\right)=L
$$

and is also a viscosity supersolution of

$$
\partial_{t} u-\frac{1}{2} \operatorname{tr}\left(\sigma(t) \sigma(t)^{\top} \nabla^{2} u\right)=-L,
$$

in both cases on $\mathbb{R}^{N} \times(0, T)$, and for some $L>0$ big enough and independent of $t$. Then fix $s \in[0, T)$ and consider the functions

$$
\begin{aligned}
& \bar{v}(x, t)=u(x, s)+C(t-s), \\
& \underline{v}(x, t)=u(x, s)-C(t-s) .
\end{aligned}
$$

These are respectively a viscosity supersolution of (5.1) and a viscosity subsolution of (5.2) on $\mathbb{R}^{N} \times[s, T)$, if $C$ is big enough. $C$ depends only on the $\mathbb{W}^{2, \infty}$ norm of $u$ and is independent of both $t$ and $s$. Therefore, by the comparison for the equations (5.1), (5.2), which follows by standard arguments (see [6]), we get

$$
u(x, s)-C(t-s) \leq u(x, t) \leq u(x, s)+C(t-s) .
$$

That gives the Lipschitz continuity in time.

By Theorem 3.4 and Theorem 5.1, then easily follows Theorem 3.5.

Acknowledgements. The author is grateful to Prof. P. Cannarsa and Prof. C. Sinestrari for valuable comments. He would like also to thank the anonymous referee for the careful reading of the manuscript and helpful suggestions.

\section{REFERENCES}

[1] G. Barles and B. Perthame, Comparison principle for Dirichlet-type Hamilton-Jacobi equations and singular perturbation of degenerated elliptic equations. Appl. Math. Optim., 21:21-44, 1990.

[2] G. Barles, Solutions de Viscositè des Èquations de Hamilton-Jacobi. Mathématiques et Applications, Springer, Paris, 1994.

[3] M.G. Crandall, K. Fok, M. Kocan, and A. Swiech, Remarks on Nonlinear Uniformly Parabolic Equations. Indiana Univ. Math. J., 47, 1998.

[4] M.G. Crandall, P.L. Lions, Quadratic growth of solutions of fully nonlinear second order equations in $\mathbb{R}^{m}$. Differential Integral Equations, 3:601-616, 1990. 
[5] M.G. Crandall, H. Ishii, The maximum principle for semicontinuous functions. Differential Integral Equations, 3:1001-1014, 1990.

[6] M.G. Crandall, H. Ishii, and P.L. Lions, User's guide to viscosity solutions of second order partial differential equations. Bull. Amer. Math. Soc., 27:1-67, 1992.

[7] D. Duffie, Dynamic Asset Pricing Theory. Princeton University Press, New Jersey, 1996.

[8] X. Gabaix, and O. Vigneron, The valuation of Mortgage-Backed Securities: theory and evidence. Harvard University, University of Chicago, 1998.

[9] Y. Giga, S. Goto, H. Ishii, and M.H. Sato, Comparison principle and convexity preserving properties for singular degenerate parabolic equations on unbounded domains. Indiana Univ. Math. J., 40:444-469, 1991.

[10] H. Ishii, On uniqueness and existence of viscosity solutions of fully nonlinear second-order elliptic PDEs. Comm. Pure Appl. Math., 42:15-45, 1989.

[11] H. Ishii, and K. Kobayasi, On the uniqueness and existence of solutions of fully nonlinear parabolic PDEs under the Osgood type condition. Differential Integral Equations, 7:909920, 1994.

[12] H. Ishii, and P.L. Lions, Viscosity Solutions of Fully Nonlinear Second-Order Elliptic Partial Differential Equations. J. Differential Equations, 83:26-78, 1990.

[13] R. Jensen, P.L. Lions, and P.E. Souganidis, A uniqueness result for viscosity solutions of second order fully nonlinear partial differential equations. Proc. Amer. Math. Soc., 102:975-978, 1987.

[14] I. Karatzas, and S. Shreve, Brownian Motion and Stochastic Calculus. Springer Verlag, NewYork, 1998.

[15] I. Karatzas, and S.E. Shreve, Methods of Mathematical Finance. Springer Verlag, New York, 1998.

[16] N.V. Krilov, Controlled Diffusion Processes. Springer Verlag, New York, 1980.

[17] M. Papi, Analysis of a Financial Model for Valuing Mortgage-Backed Securities. Master Thesis, Istituto per le Applicazioni del Calcolo (IAC-CNR), Italy, Rome, 1999.

[18] M. Papi, A generalized Osgood condition for viscosity solutions to fully nonlinear parabolic degenerate equations. Adv. Differential Equations., 7(9):1125-1151, 2002.

[19] R. Stanton, Rational prepayment and the valuation of Mortgage-Backed Securities. The Review of financial studies, 8:677-708, 1995. 